\title{
An Approach to measure the performance metrics of IEEE architectures with and without Relay nodes
}

\author{
Fahmina Taranum ${ }^{1}$, Khaleel Ur Rahman Khan ${ }^{2}$
}

Osmania University: Computer Science and Engineering ${ }^{1}$, J.N.T.U.H.: Computer Science and Engineering ${ }^{2}$

Muffakham Jah College of Engineering and Technology ${ }^{1}$, ACE Engineering College ${ }^{2}$, Hyderabad, India

${ }^{1}$ ftaranum@mjcollege.ac.in, ${ }^{2}$ khaleelrkhan@gmail.com

\section{Received: 10 ${ }^{\text {th }}$ Feb 2018, Accepted: 18 $^{\text {th }}$ March 2018, Published: 30 $^{\text {th }}$ April 2018}

\begin{abstract}
The recent enhancements in signal processing techniques have led to faster and efficient mode of communication. In today's smart environment, people with smart devices (nodes) can freely organize and configure scenarios to receive and send packets to destination over multiple nodes via intermediate connectives. The paper portrays multiple scenarios with and without relay node for both IEEE 802.11 and IEEE 802.16 architectures along with performance metrics of both. The architecture was designed using fewer mobile nodes and one base station which is connected via subnet .The CBR traffic is activated from the base station to the mobile stations and the traffic flows from base station to subscriber stations acting as destination nodes. The performance metrics parameter evaluated and analysed which includes average transmission delay, utilization factor, Average signal power, interference and path loss.
\end{abstract}

Keywords: IEEE 802.11, IEEE802.16, Base station (BS), Subscriber station (SS) or Mobile station (MS), Constant bit rate (CBR)

\section{Introduction}

The major concern in current technological advancement is power optimization, minimizing the battery usage, maximizing the features at the MSs. As the mobile stations moves with high speed the battery utilization is also high, which is taken as a challenge and many attempts were made to reduce the power consumption when MSs communicate with the BSs. The analysis shows that the signal arrival power at the mobile station is more when compared to base station in the designed scenarios. The concept of selecting a station type as that of base and subscriber type is available only in IEEE 802.16 but not in 802.11 , still the scenario can be designed as that of destination and source with CBR connecting the source and destination node in IEEE 802.11.The closer the placement of node the better is the transmission. The provision to enable OFDMA at the MAC layer is available in IEEE 802.16 only. Therefore the OFDMA burst received and forwarded to MAC is analysed only in IEEE 802.16.To model the PHY layer certain characteristics need to be incorporated at the source and the destination end like modulation, coding, noise, interference and antenna gains. In
QualNet, Physical Layer model consists of - a PHY and an antenna component. The PHY component caters for signal transmission, reception and reflects the effects of the MAC, distortions and interference. The antenna component models the functions and properties of the antenna like the model of antenna, etc.

The rest of the paper is organized as follows: section II provides a brief description of related work; section III describes about topologies, section IV discusses the result analysis of 802.11 and 802.16 with and without relay. Section $\mathrm{V}$ portrays conclusion and future research scope.

\section{Related Work}

Paper [1] is documented on the upshot of power optimization in Mobile stations. Different levels of power were taken as input using user defined energy model at the base station, mobile station and relay station with different modulation techniques from base to relay and relay to mobile station for reducing the energy level consumption at the mobile nodes during transmission.

Paper [2] is about the routing algorithms, energy models, battery models and propagation models. The different energy models discussed include user defined, Mica motes, Micaz and Generic collaborated with linear battery model and two ray propagation model. A comparative study was made for routing algorithms like AODV, DYMO and DSR for measuring throughput, Average Jitter, End to End Delay and energy consumption.

Paper [3] discuses the comparison of Genetic Algorithm and Simulated Annealing meta-heuristics to obtain the minimum energy consumed in ad-hoc wireless networks. The signal power requirements are selected from this paper to compare the path loss and interference in designed scenario.

Paper[4] portray the antenna models for measuring the QoS parameters like Average Throughput, Average End to End Delay, Average Jitter, and Packet Delivery Ratio were evaluated to check the performance and efficiency of the network in different scenarios. This analysis was based on omni directional, steerable and switched beam antennas were used for all nodes in the network. The idea to 
measure performance metrics is selected from this paper.

\section{Topologies}

A. Existing topology

In this scenario the continental network topologies with high mobility environment are considered. The mobility model of IEEE 802.11 with no relay node is shown in Fig. 1 to Fig. 3. Node 7 is acting as base station and node 1,2 and 3 are acting as subscriber station which moves along the random path set using random waypoint model denoted by red flags as shown in table II. Node 7 is connected to mobile nodes using a subnet and the traffic is regulated from base to subscriber station using CBR (represented by Green line).

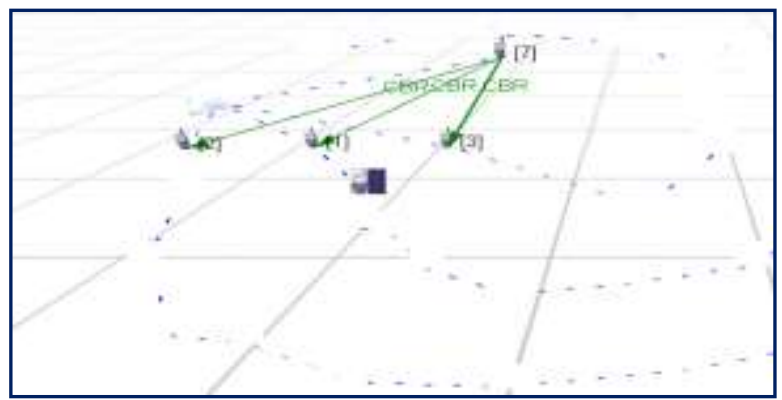

Fig. 13 -D view of transmission from base $\&$ mobile stations using 802.11

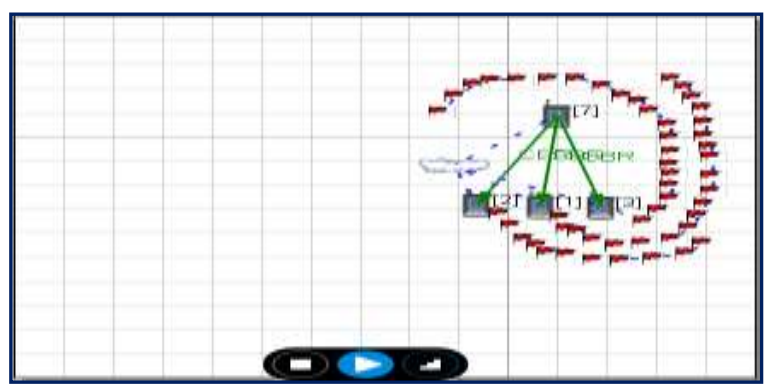

Fig. 2 Transmission from base to three mobile stations using 802.11

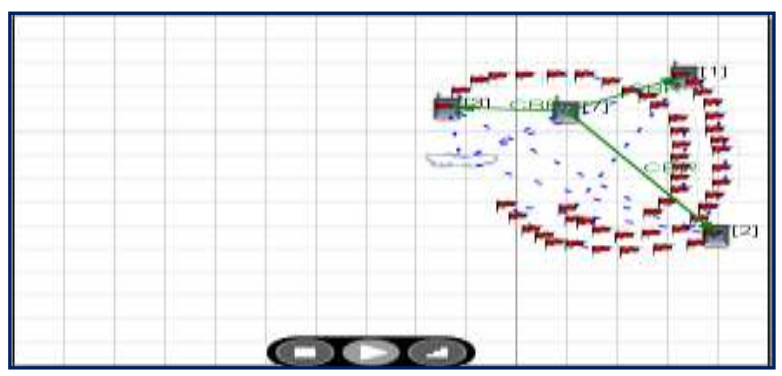

Fig.3 Nodes final position after mobility
Table I

NETWORK PARAMETERS FOR EXISTENT
NETWORK TOPOLOGIES
\begin{tabular}{|l|l|}
\hline \multicolumn{1}{|c|}{ Parameter } & \multicolumn{1}{c|}{ Value } \\
\hline Qualnet version & 7.4 \\
\hline Terrain Size & $1500 * 1500$ meters \\
\hline Physical Layer & $\begin{array}{l}802.11 \mathrm{~b} \text { Radio /802.16 } \\
\text { Radio }\end{array}$ \\
\hline Mobility model & Random waypoint \\
\hline Fading Model & Ricean Fading Model \\
\hline Traffic Type & CBR \\
\hline Network Layer & IPv4 \\
\hline Routing Protocol & AODV \\
\hline Simulation Time & 60 seconds \\
\hline Frequencies & 2.4 GHz \\
\hline No of Channels & 2 (one hop) or 1 (no hop) \\
\hline $\begin{array}{l}\text { Gaussian } \\
\text { component file }\end{array}$ & default.SR10000.fading \\
\hline
\end{tabular}

The parameters used in the network scenario is depicted in table I. Table II shows the random waypoint created manually for the three different paths for mobile station 1, 2 and 3 with the $\mathrm{X}-\mathrm{Y}$ position at every second of simulation time. It can be further noted that the relay station (node 8 ) and base station (node 7) will have a fixed Position in $\mathrm{X}-\mathrm{Y}$ axis of table II.

Table II: Random-waypoint model for Mobility

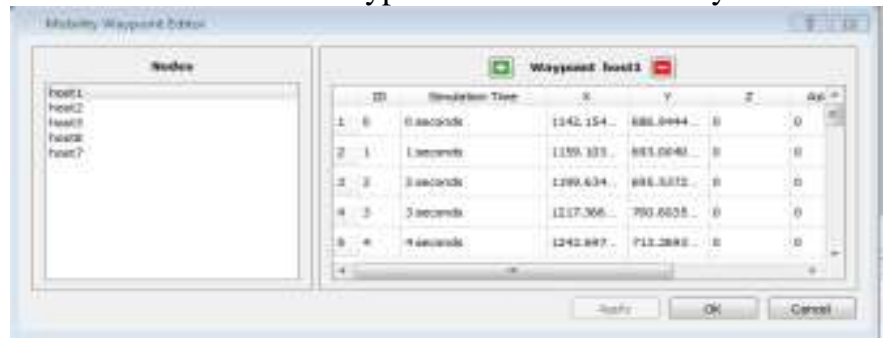

\section{B. Single Hop Relay Strategy}

Single hop is added to existing architecture as shown in fig.4 and fig. 5 for the transmission from base station 7 to relay node 8 and then with this single hop it is further transmitted to mobile nodes using IEEE 802.11 viz node 1,2 , and 3.The relay node acts as a caching node, which is an intermediate node connecting a source and destination node in wireless network used to store and transmit the data. 


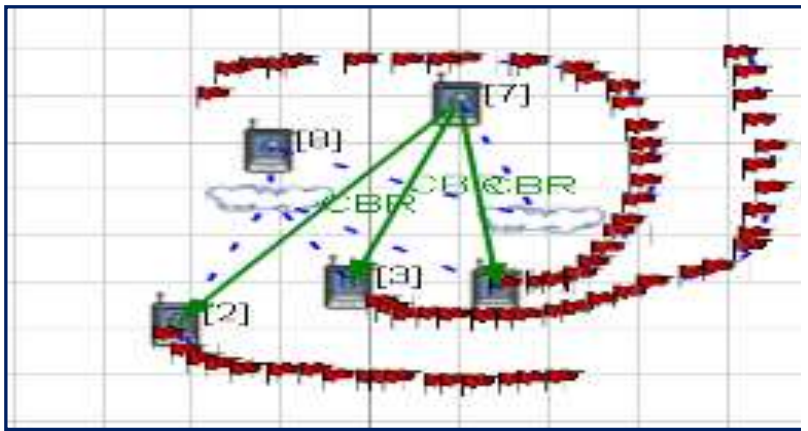

Fig. 4 Transmission using IEEE 802.11 with relay

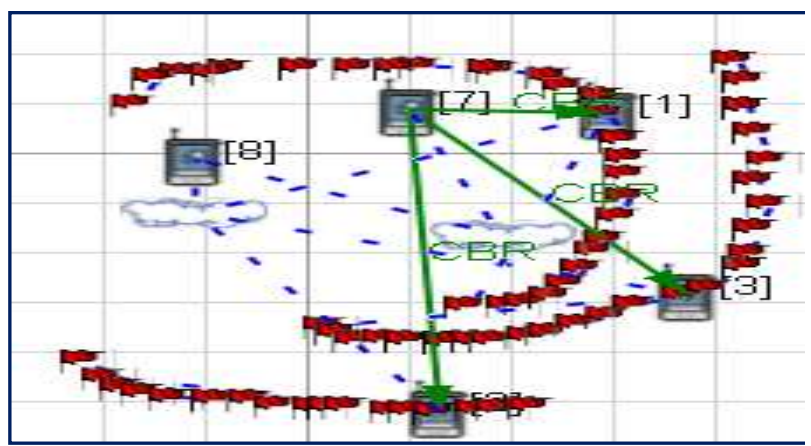

Fig. 5 Nodes final position after mobility

As can be seen in fig. 5, mobile node moves along the random path indicated by red colour flags till it reaches the final position indicated by the last flag in the path to stop. The relays transmit the data accordingly to the mobile stations which are under its purview.

C. Scenario using 802.16 without relay

In IEEE 802.16 the station type property is available to select the station as of the type base or subscriber. The physical layer protocol is set to 802.16 with association type enabled and Mac layer 802.16 is configured.

Fig 6 depicts one such scenario.

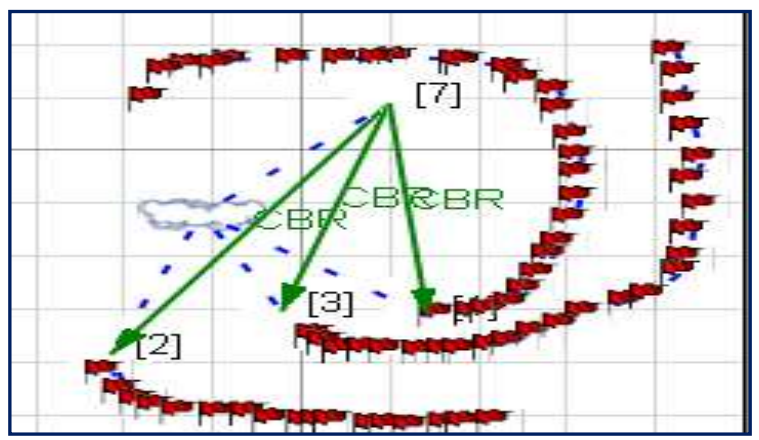
802.16

Fig. 6 Transmission without Relay node using

The blue dotted line shows the connectivity of node $1,2,3$ to the subnet 192.0.3.1.Three CBR links are for one source and three destination nodes in which the data is transmitted using AODV routing protocol.

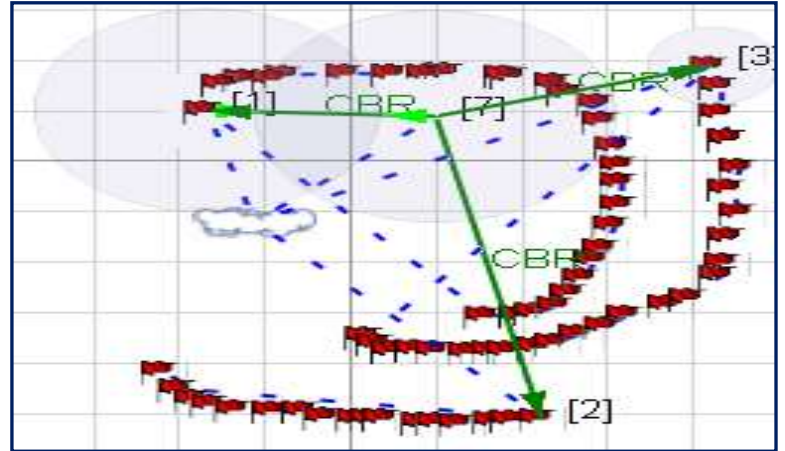

Fig. 7 Transmission and mobility without Relay node using 802.16

The shaded circles around the node 1, 3 and 7 are the transmitting regions. The light green arrow head shows the direction of packet transmission in between node 1 to node 7 as shown in fig. 7 .

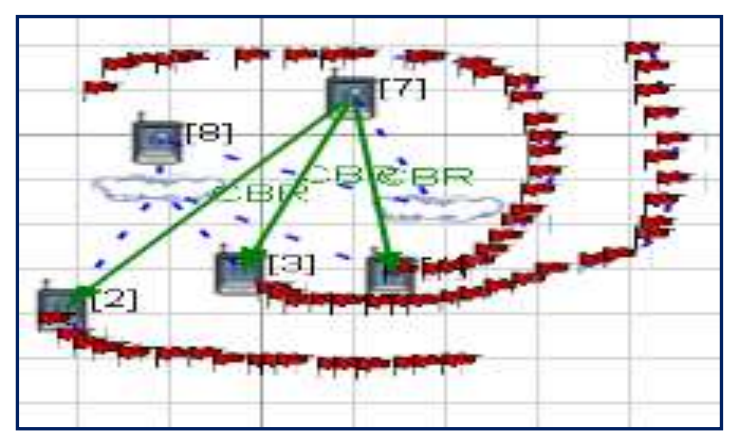

Fig. 8 Transmission with Relay node using 802.16

A relay node is being added in between base and mobile stations connected by a subnet with an address 190.0.3.2 while the relay and base are connected with a subnet with address 190.0.3.1 as shown if Fig. 8.As node 2 is far apart from the base station it receive the least number of packets.

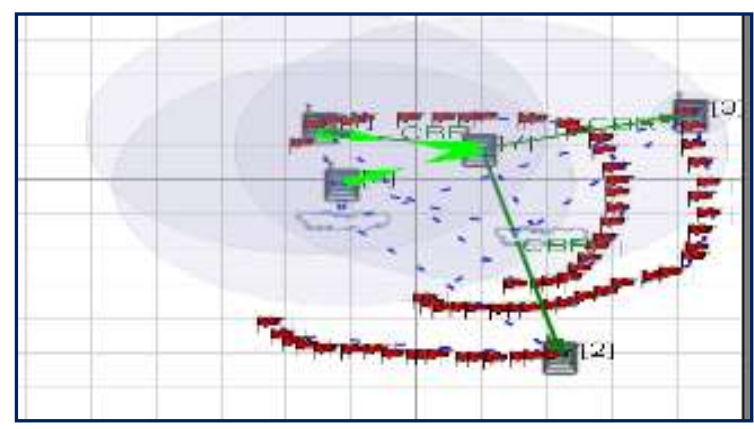

Fig. 9 Transmission and mobility with Relay node using 802.16

The packet is being transmitted in between base, relay and mobile stations as depicted in fig 9 and fig. 10 . 


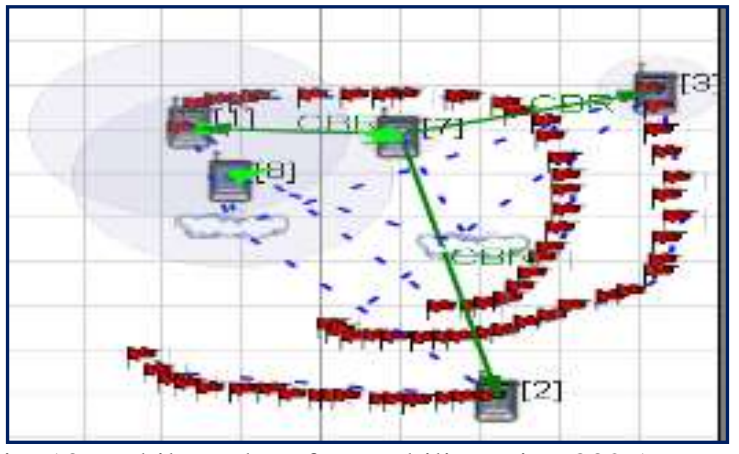

Fig. 10 Mobile nodes after mobility using 802.16

After running the simulation the results shows the default address assigned to the nodes. The reason for taking less number of nodes in the scenario is to ease out the comparison parameters used to measure the performance metrics. Table III shows the network layer scenario generated after running the simulation

Tables III: Network Address, type and node id used in the scenario

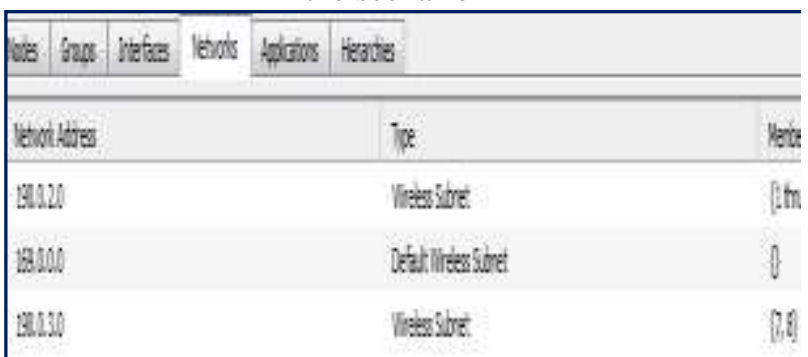

The nodes are assigned addresses as can be seen from table IV in which the node 1, 2, 3, 7 and 8 are assigned address190.0.2.2, 190.0.2.1, 190.0.2.4 and 190.0.3.2 and 190.0.2.5 respectively. It also shows the routing protocol been used in $\mathrm{t}$ he scenario as AODV.

Tables IV

Interface details generated in the scenarios

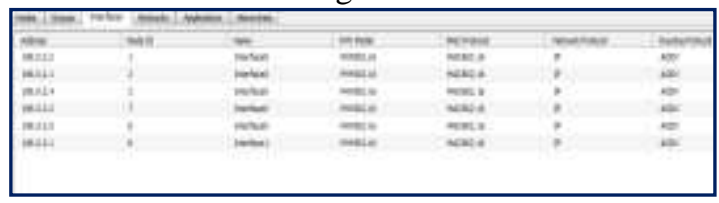

\section{Results and Discussion}

The analysis of the four different simulation scenarios generated using the designed model for IEEE 802.11 no relay and relay, IEEE 802.16 no relay and relay are shown in table V to table VIII. The Fading model is set to Ricean fading model as it is used for scenarios in which the sight signal is dominant signal seen at the receiver and for line of sight communication. The unit of time, average interference used in the modeling is seconds and $\mathrm{dBm}$ respectively.
Table V: Physical layer analysis of IEEE 802.11 No-Relay

\begin{tabular}{|l|l|l|l|l|l|}
\hline Node Id & \multicolumn{1}{|c|}{$\mathbf{1}$} & \multicolumn{1}{|c|}{$\mathbf{2}$} & \multicolumn{1}{|c|}{$\mathbf{3}$} & \multicolumn{1}{|c|}{$\mathbf{7}$} & \multicolumn{1}{c|}{$\mathbf{8}$} \\
\hline Signal transmitted & 3 & 3 & 43 & 64 & 5 \\
\hline Signal detected & 120 & 120 & 80 & 59 & 118 \\
\hline Signal sent to MAC & 4 & 3 & 46 & 39 & 28 \\
\hline $\begin{array}{l}\text { Time spent } \\
\text { transmitting }\end{array}$ & 0.001344 & 0.001344 & 0.0156 & 0.064664 & 29 \\
\hline $\begin{array}{l}\text { Time spent } \\
\text { receiving }\end{array}$ & 0.00224 & 0.001793 & 0.0646 & 0.018872 & 0.067928 \\
\hline $\begin{array}{l}\text { Avg. transmitting } \\
\text { delay }\end{array}$ & .000000458 & .000000464 & 000000627 & .000000632 & .000000639 \\
\hline Utilization & 0.00006 & 0.000053 & 0.0001345 & 0.001393 & .001171 \\
\hline Avg. signal power & -75.193 & -75.427 & -77.56 & -77.807 & .538916 \\
\hline Avg. interference & -90.97 & -90.97 & -90.97 & -90.97 & -90.970077 \\
\hline Avg. Path loss & 86.72 & 86.76 & 89.526 & 89.587 & 75.306 \\
\hline & & & & & \\
\hline
\end{tabular}

Tables VI

Physical layer analysis of IEEE 802.11 Relay

\begin{tabular}{|c|c|c|c|c|}
\hline Node Id & 1 & 2 & 3 & 7 \\
\hline \begin{tabular}{|l|} 
Signal \\
transmitted
\end{tabular} & 19 & 3 & 3 & 36 \\
\hline $\begin{array}{l}\text { Signal } \\
\text { detected }\end{array}$ & 42 & 58 & 58 & 25 \\
\hline \begin{tabular}{|l|} 
Signal sent \\
to MAC \\
\end{tabular} & 16 & 1 & 3 & 12 \\
\hline $\begin{array}{l}\begin{array}{l}\text { Time spent } \\
\text { transmitting }\end{array} \\
\end{array}$ & 0.007744 & 0.00135 & 0.001344 & 0.02369 \\
\hline \begin{tabular}{|l|} 
Time spent \\
receiving
\end{tabular} & 0.019368 & 0.00135 & 0.00224 & 0.0064 \\
\hline \begin{tabular}{|l|} 
Avg. \\
transmitting \\
delay \\
\end{tabular} & 0.00000063 & 0.000000732 & 0.000000614 & 0.000000633 \\
\hline Utilization & 0.000454 & 0.000045 & 0.00006 & 0.000504 \\
\hline \begin{tabular}{|l|} 
Avg. signal \\
power
\end{tabular} & -78.085 & -80.439 & -77.839 & -77.56 \\
\hline \begin{tabular}{|l|} 
Avg. \\
interference
\end{tabular} & -90.97 & -90.97 & -90.97 & -90.97 \\
\hline $\begin{array}{l}\text { Avg. Path } \\
\text { loss }\end{array}$ & 89.52 & 90.88 & 89.236 & 80.59 \\
\hline
\end{tabular}

It can be seen from table $\mathrm{V}$ that the time spent in transmission at the base node is quite high when compared to all the mobile nodes. Average path loss is high at node 2 as it is at a farther distance from the base station. The rate of signal transmitted, utilization and average signal power is more at the base station.

The Average interference and the noise ratio remain same in both the IEEE scenarios. The average signal power required is high at the relay node when compared to other nodes in the architecture. The physical layer utilization is high in relay node when compared to base node as shown in table VI. 
Tables VII: Physical layer analysis of IEEE 802.16 No-Relay

\begin{tabular}{|l|c|c|c|c|}
\hline NodeId & $\mathbf{1}$ & $\mathbf{2}$ & $\mathbf{3}$ & $\mathbf{7}$ \\
\hline & & & & \\
\hline Signal transmitted & 752 & 0 & 537 & 3000 \\
\hline $\begin{array}{l}\text { Signal received \& forwarded } \\
\text { to MAC }\end{array}$ & 2968 & 0 & 2109 & 1289 \\
\hline Signal locked on by PHY & 2968 & 0 & 2109 & 1289 \\
\hline $\begin{array}{l}\text { OFDMA burst received and } \\
\text { forwarded to MAC }\end{array}$ & 6151 & 0 & 4374 & 1289 \\
\hline Avg. Pathloss & 100 & 0 & 101.4 & 96 \\
\hline Signal arrival(power) & $\begin{array}{c}2.02 \\
\text { E-08 }\end{array}$ & $\begin{array}{c}3.71 \\
\text { E-10 }\end{array}$ & $\begin{array}{c}7.78 \\
\text { E-09 }\end{array}$ & 2.02 \\
E-08 \\
\hline
\end{tabular}

The interpretations made from Table VII shows that the entire signal received are forwarded to MAC are locked by physical layer. The value 0 in node 2 indicates that there is no transmission via node 2 as it is not reachable from the base station since it is at a farther location, which is out of scope.

Tables VIII: Physical layer analysis of IEEE 802.16 Relay

\begin{tabular}{|l|l|l|l|l|l|l|}
\hline Node Id & $\mathbf{1}$ & $\mathbf{2}$ & $\mathbf{3}$ & $\mathbf{7}$ & $\mathbf{8 . 1}$ & 8.2 \\
\hline signal transmitted & 753 & 0 & 537 & 3000 & 742 & 743 \\
\hline $\begin{array}{l}\text { signal received \& } \\
\text { forwarded to MAC }\end{array}$ & 2968 & 0 & 2109 & 2770 & 2997 & 2961 \\
\hline $\begin{array}{l}\text { signal locked on by } \\
\text { PHY }\end{array}$ & 2968 & 0 & 2109 & 2771 & 2997 & 2961 \\
\hline $\begin{array}{l}\text { OFDMA burst } \\
\text { received and } \\
\text { forwarded to MAC }\end{array}$ & 6042 & 0 & 4284 & 2770 & 6107 & 6034 \\
\hline AVG pathloss & 95.83 & 0 & 102.2 & 94.06 & 94.229 \\
\hline $\begin{array}{l}\text { SIGNAL } \\
\text { ARRIVAL(POWER) }\end{array}$ & $2.02 \mathrm{E}-$ & 08 & $0.00 \mathrm{E}+00$ & $\begin{array}{l}7.78 \mathrm{E}-4.81 \mathrm{E}-3.46 \mathrm{E}-3.46 \mathrm{E}-109 \\
08\end{array}$ \\
\hline
\end{tabular}

The Ricean fading effects for the modelled scenario works with the value given next to the variable Sampling-rate 10000

Base-Doppler-frequency 30.0

Number-of-Gaussian-components $=163840$

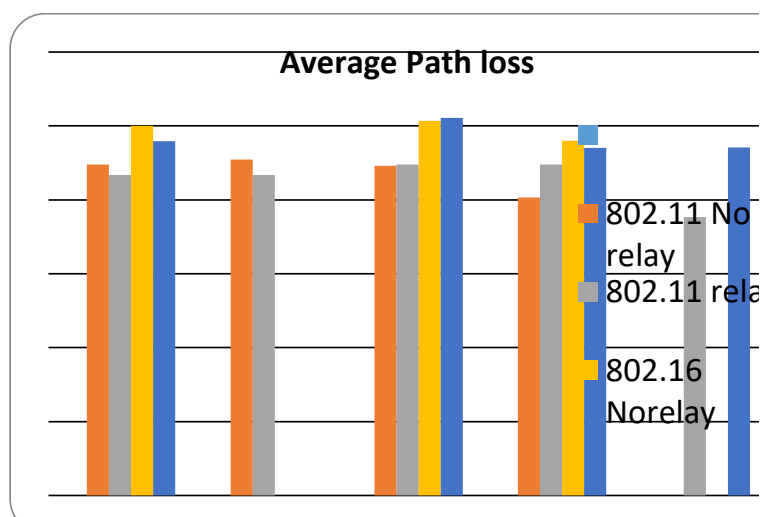

Fig. 11 Average path loss of all the designed scenarios

Fig 11 demonstrates that the path loss is more in IEEE 802.11 architecture when compared to IEEE 802.16.

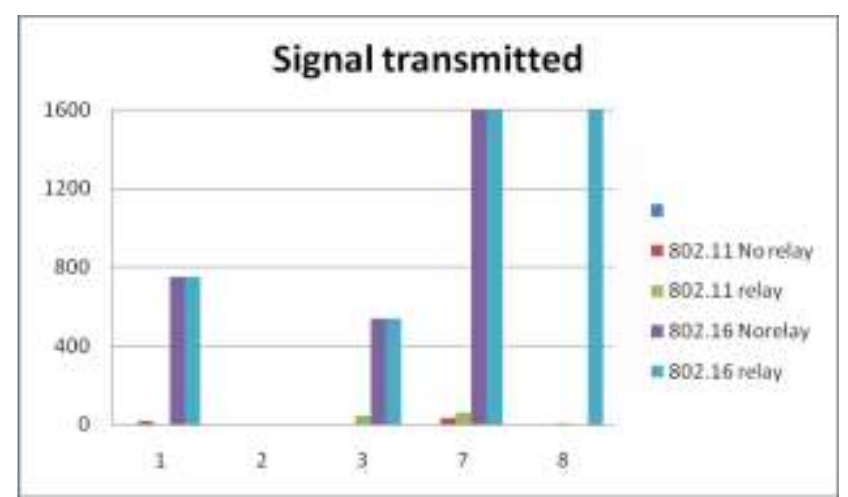

Fig. 12 Signal transmitted for all the designed scenarios

The rate of signal transmitted is depicted in fig 12.The final conclusion that can be made from the analysis is IEEE 802.16 without relay outperform IEEE 802.16 with relay.

\section{Conclusion}

The analysis shows that the performance is best in case of single relay node network when compared to no relay as depicted in Fig. 11. The same node positions and connectivity are used to compare the architecture on same data set for all the designed scenarios.Under future scope the mathematical evaluation can be used to show the power consumption levels of mobile and base station and design a scenario which utilizes less power using different energy models, battery models and power aware routing algorithms.

\section{References}

[1]. Akshay Jain," Reduction of Power Consumption at Mobile Station in Mult hop Networks in mobile Environments,2012 1st International Conference on Emerging Technology Trends in Electronics, Communication and networking.

[2]. Bharti Soni, Mehajabeen Fatima," Overview of Energy Consumption and Propagation in MANET", International Journal of Innovative Research in Science and Technology Vol. 6, Issue 7, July 2017

[3]. Ibukunola. A. Modupea, Oludayo. O. Olugbarab, Abiodun Modupec, Minimizing Energy Consumption in Wireless Ad hoc Networks with Meta heuristics, The 4th International Conference on Ambient Systems, Networks and Technologies, 2013

[4]. Marco Fotino, Salvatore Marano, "EEOLSR: Energy Efficient OLSR Routing Protocol for Mobile Adhoc Networks," IEEE Transactions, pp. 1-7, 2008

[5]. J. Kanjanarot, K. Sitthi, and C \& Saivichit, "Energy-based Route Discovery Mechanism in Mobile Ad Hoc Networks," ICA0T2006, 2006 
[6]. Ashish Kumar, M. Q. Rafiq, and Kamal Bansal, "Performance evaluation of energy consumption in MANET," International Journal Of Computer Applications, vol. 42, no. 2, pp. 7-12, 2012

[7]. S. Gopinath, Dr. A. Rajaram and N. Suresh Kumar, "Improving minimum energy consumption in ad hoc networks under different scenarios," International Journal of Advanced and Innovative Research(IJAIR), pp. 40-46, 2012

[8]. Gitanjali Chandwani, Soumendra Nath Datta, Saswat Chakrabarti, "Relay Assisted Cellular System for Energy Minimization", Annual IEEE Indian Conference (INDICON), 2010 